\title{
Para-kala-azar dermal leishmaniasis in a patient in Brazil: a case report
}

\author{
José Angelo Lauletta Lindoso[ ${ }^{[1],[2], ~ C a r l o s ~ H e n r i q u e ~ V a l e n t e ~ M o r e i r a ~}{ }^{[2],[3],[6],}$ \\ Beatriz Julieta Celeste ${ }^{[3]}$, Luiza Keiko Matsuka Oyafuso ${ }^{[2],}$ \\ Pedro Moreira Folegatti ${ }^{[4]}$ and Eduard Evert Zijlstra ${ }^{[5]}$
}

\begin{abstract}
[1]. Laboratório de Soroepidemiologia (LIM 38), Hospital das Clínicas da Faculdade de Medicina da Universidade de São Paulo, São Paulo, SP, Brasil. [2]. Instituto de Infectologia Emilio Ribas, São Paulo, SP, Brasil.

[3]. Instituto de Medicina Tropical da Universidade de São Paulo, São Paulo, SP, Brasil.

[4]. The Jenner Institute, Nuffield Department of Medicine, University of Oxford, United Kingdom.

[5]. Rotterdam Centre for Tropical Medicine, Rotterdam, the Netherlands.

[6]. Laboratório de Parasitologia (LIM 46), Hospital das Clínicas da Faculdade de Medicina da Universidade de São Paulo, São Paulo, SP, Brasil.
\end{abstract}

\begin{abstract}
Visceral leishmaniasis is common in Brazil and is caused by Leishmania (Leishmania) infantum/chagasi. Post-kala-azar dermal leishmaniasis frequently follows visceral leishmaniasis caused by L. donovani, and para-kala-azar dermal leishmaniasis refers to an uncommon presentation wherein it occurs simultaneously along with visceral leishmaniasis. While post-kala-azar dermal leishmaniasis only occurs occasionally in L. infantum/chagasi infections, it frequently occurs in patients with concomitant immunosuppression (HIV co-infection). Here, we describe the first case of para-kala-azar dermal leishmaniasis in Brazil. It is important to raise awareness of post- and para-kala-azar dermal leishmaniasis in L. infantum endemic areas as these patients may contribute to visceral leishmaniasis transmission.
\end{abstract}

Keywords: Visceral leishmaniasis. Dermal leishmaniasis. Leishmania infantum.

\section{INTRODUCTION}

Visceral leishmaniasis (VL) is common in South America and $90 \%$ of cases occur in Brazil. The main vector is Lutzomyia longipalpis and canines function as the main reservoir ${ }^{1}$. In recent years, the disease has spread to all regions and emerged in urban areas ${ }^{2}$. In Latin America, cutaneous manifestations in leishmaniasis are related to Leishmania from the subgenus Viannia ${ }^{3}$; however, there are reports of cutaneous lesions similar to those observed in post-kala-azar dermal leishmaniasis (PKDL), caused by Leishmania infantum, mainly in human immunodeficiency virus (HIV)-infected patients ${ }^{4,5}$. In addition, L. infantum can cause atypical cutaneous leishmaniasis in Central America unrelated to $\mathrm{VL}^{6}$. Here, we describe an HIVnegative patient with VL with an unusual clinical course and outcome, in which a relapse of VL was accompanied by a PKDL-like skin rash.

Corresponding author: Dr. José Angelo Lauletta Lindoso.

e-mail: jlindoso@usp.br

Received 23 November 2016

Accepted 1 December 2017

\section{CASE REPORT}

A 36-year-old farmer presented at the Public General Hospital in Santos City because of fever. He was born in the city of Sao Vicente in South-East Brazil. He reported that he had worked in Joao Pessoa, Paraíba, North-East Brazil, an endemic area for VL, from 2000 to 2006. During that time, his dog became ill and died. He first presented in November 2006 with a long history of daily fever, headache, general weakness, and dizziness, and he reported to have lost weight (18kg in the last 3 months). His weight at the time of admission was $53 \mathrm{~kg}$. Hepatosplenomegaly was noted on physical examination. VL was suspected and confirmed by a bone marrow aspirate that showed Leishmania amastigotes. HIV test results were negative. He was treated with meglumine antimoniate (Glucantime) intramuscular (IM) in a dose of $20 \mathrm{mg} / \mathrm{kg} /$ day for 28 days with positive clinical response.

He did not return for follow-up but presented in January 2008 with a (clinically diagnosed) relapse and was treated with two cycles of Abelcet (amphotericin B lipid complex) daily $(1 \mathrm{mg} / \mathrm{kg} / \mathrm{day})$ for 20 days followed by two cycles of glucantime $(20 \mathrm{mg} / \mathrm{kg} /$ day for 28 days) combined with Abelcet $(1 \mathrm{mg} / \mathrm{kg} /$ day). In March 2008, he was admitted to the Institute of Infectology Emilio Ribas at São Paulo with fever and hepatosplenomegaly; a second relapse of VL was suspected. 
Anti-leishmania antibodies were demonstrated in the immunofluorescence test (immunoglobulin (Ig)G 1:160) and enzyme-linked immunosorbent assay (ELISA) (IgG 1: 1280); the Montenegro test results were negative. He was treated according to the National Guidelines for treatment of VL with liposomal amphotericin B (LAmb) (3 $\mathrm{mg} / \mathrm{kg}$ once daily for 7 days) and exhibited a positive clinical response.

Five months later, he developed diarrhea and vomiting and he had lost $5 \mathrm{~kg}$ of weight. On physical examination, he was dehydrated, and his weight was $48 \mathrm{~kg}$. In addition, on the skin of the chest and abdomen, erythematous macules and papules (diameter $0.5-1.0 \mathrm{~cm}$ ) were noted (Figure 1A and Figure 1B). He was re-admitted to the hospital and received intravenous (IV) fluid replacement. A bone marrow aspirate showed numerous Leishmania amastigotes; in addition, a skin biopsy demonstrated an inflammatory process characterized by numerous small, oval, plasmacytoid structures indicative of infection by Leishmania sp. Additionally, on the surface, the epidermis was thin, with fibrosis of the papillary dermis.

The immunofluorescence test for Leishmania showed an IgG titer of 1:64, whereas in the ELISA, the IgG titer was 1:320. Treatment was initiated with amphotericin B deoxycholate $(50 \mathrm{mg} /$ day for 4 days), followed by LAmb (3 $\mathrm{mg} / \mathrm{kg}$ for 10 days), with positive response.

Laboratory investigations showed negative results for antibodies against HIV, human T-cell lymphotropic virus (HTLV)-1 and -2, T. cruzi, and hepatitis A. IgG antibodies were found against cytomegalovirus (CMV) and toxoplasma. The IgM ELISA results for schistosomiasis were positive. Immunological assessment revealed the following: cluster of differentiation CD4+count , 777 cells $/ \mathrm{mm}^{3}$; CD8+count, 633 cells $/ \mathrm{mm}^{3}$; and CD4/CD8 ratio: 1.23. Lymphocyte proliferation tests showed proliferation on stimulation with Leishmania and Toxoplasma antigen. Proliferative responses against mitogens were normal.

Secondary prophylaxis was suggested, but he did not return for follow-up. Ten months later, he presented with fever and cervical lymphadenopathy that initiated 20 days earlier; a cervical lymph node biopsy showed its architecture replaced by hard granulomas without central necrosis and rare giant cells. In addition, in the macrophages, a large amount of Leishmania $s p$. was observed.

There was no evidence for hepatosplenomegaly. He was treated with LAmb $4 \mathrm{mg} / \mathrm{kg} /$ day for 7 days and secondary prophylaxis was initiated with pentavalent antimonial (IV or IM) (850 mg every 28 days), after which he remained well during 9 months of follow-up. To better characterize treatment response observed after treatment and secondary prophylaxis, we measured anti-Leishmania antibody titers using ELISA and indirect immunofluorescence (IFI), using Leishmania major-like total antigen; decreases in titers were observed 6 and 16 months post-treatment (Figure 2).

\section{DISCUSSION}

In this patient, inflammatory processes associated with fibrosis from papillary dermis with the presence of Leishmania was observed in cutaneous lesions. In macular lesions of PKDL, chronic inflammation is characterized by infiltrates

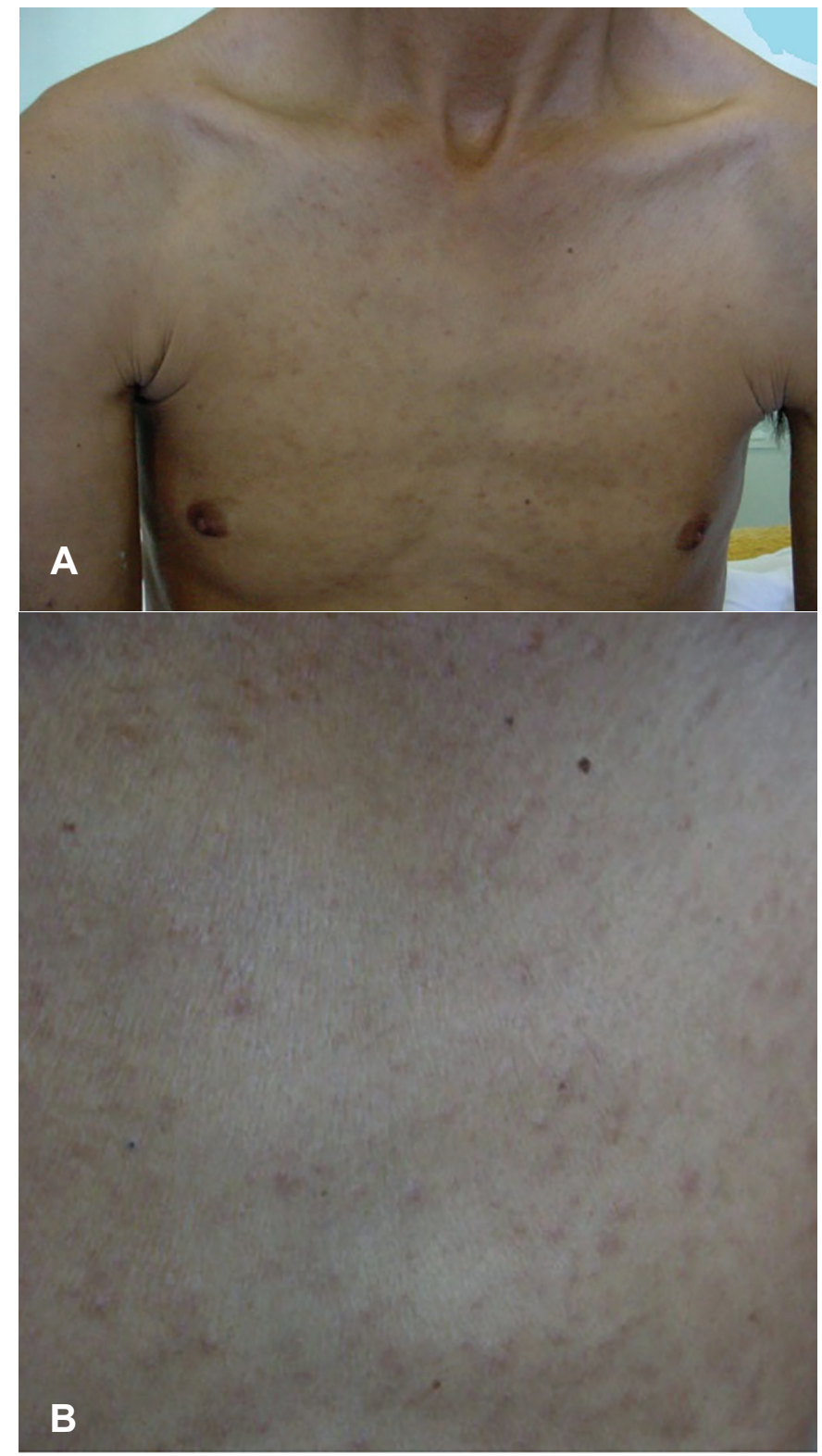

FIGURE 1: (A): Presence of papules in the trunk, caused by Leishmania infantum, on a patient who presented with visceral leishmaniasis. (B): Details of the lesions (papules) in the trunk.

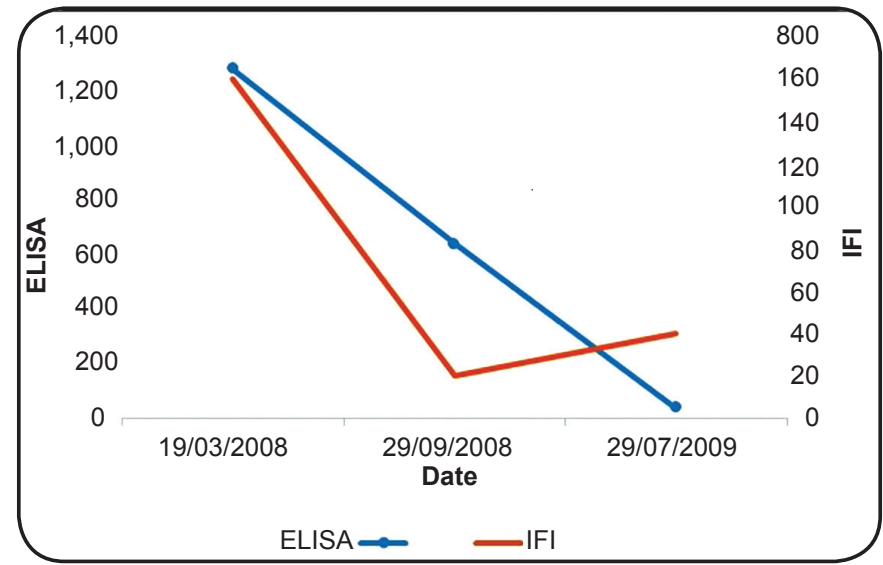

FIGURE 2: Level of anti-Leishmania antibodies measured using an enzymelinked immunosorbent assay (ELISA) and indirect immunofluorescence (IFI), using total Leishmania major-like antigens, during active disease, and 6 and 16 months after treatment. 
of histiocytes, lymphocytes, and a few plasma cells, and Leishmania is also observed ${ }^{10}$. However, there are no consistent of infiltrates in PKDL lesions. The clinical manifestations of PKDL are immunologically mediated with features of a Th2 response in the skin and a systemic Th1 response, resulting in skin abnormalities in patients who are otherwise well without features of systemic leishmanial infection ${ }^{5-10,11}$. In this patient, the clinical progression from visceral disease with subsequent relapses to para-kala-azar dermal leishmaniasis and later isolated (post-kala-azar) lymphadenopathy without obvious clinical evidence of visceral disease suggests a similarly developing, but abnormal and inadequate systemic immune response $^{11-13}$. In immunosuppressed patients, in particular those who are HIVinfected, relapses are common, as some degree of developing antileishmanial immunity is needed to prevent a relapse of VL. With each relapse, the treatment becomes more difficult ${ }^{5}$. This patient had a similar clinical syndrome, which justified the decision for maintenance treatment that to date seemed to be successful. There is no consensus regarding the preferred regimen; single-dose administration of pentavalent antimonial, Lamb (AmBisome (C) or pentamidine has been used, often in cycles of 3-4 weeks.

This case illustrates the need for follow-up of patients with VL, in particular to monitor for PKDL or para-kala-azar dermal leishmaniasis as experience elsewhere indicates that as the rash often remains unnoticed and self-cures, these patients often do not report to the clinic and may play a role in transmission.

\section{Conflict of interest}

The authors declare that there is no conflict of interest.

\section{REFERENCES}

1. Romero GA, Boelaert M. Control of visceral leishmaniasis in latin america-a systematic review. PLoS Neg Trop Dis. 2010;4(1):e584.

2. Harhay MO, Olliaro PL, Costa DL, Costa CH. Urban parasitology: visceral leishmaniasis in Brazil. Trends Parasitol. 2011;27(9):403-9.
3. Goto H, Lindoso JA. Current diagnosis and treatment of cutaneous and mucocutaneous leishmaniasis. Expert Rev Anti Infect Ther. 2010;8(4):419-33.

4. Santos-Oliveira JR, Da-Cruz AM, Pires LH, Cupolillo E, Kuhls K, Giacoia-Gripp CB, et al. Atypical lesions as a sign of cutaneous dissemination of visceral leishmaniasis in a human immunodeficiency virus-positive patient simultaneously infected by two viscerotropic Leishmania species. Amer J Trop Med Hyg. 2011;85(1):55-9.

5. Zijlstra EE. PKDL and other dermal lesions in HIV co-infected patients with leishmaniasis: review of clinical presentation in relation to immune responses. PLoS Negl Trop Dis. 2014;8(11):e3258.

6. Belli A, García D, Palacios X, Rodriguez B, Valle S, Videa E, et al. Widespread atypical cutaneous leishmaniasis caused by Leishmania (L.) chagasi in Nicaragua. Amer J Trop Med Hyg. 1999;61(3):380-5.

7. Gelanew T, Amogne W, Abebe T, Kuhls K, Hailu A, Schönian G. A clinical isolate of Leishmania donovani with ITS1 sequence polymorphism as a cause of para-kala-azar dermal leishmaniasis in an Ethiopian human immunodeficiency virus-positive patient on highly active antiretroviral therapy. British J Dermatol. 2010;163(4):870-4.

8. Mendiratta V, Chander R. Post-kala-azar dermal leishmaniasis and visceral leishmaniasis in a child -or is it para-kala-azar dermal leishmaniasis? Int J Dermatol. 2009;48(9):1027-8.

9. Carvalho EM, Teixeira RS, Johnson Jr WD. Cell-mediated immunity in American visceral leishmaniasis: reversible immunosuppression during acute infection. Infect Immun. 1981;33(2):498-500.

10. Ramesh V, Singh N. A clinical and histopathological study of macular type of post-kala-azar dermal leishmaniasis. Trop Doct. 1999;29(4):205-7.

11. Ismail A, Khalil EA, Musa AM, El Hassan IM, Ibrahim ME, Theander TG, et al. The pathogenesis of post kala-azar dermal leishmaniasis from the field to the molecule: does ultraviolet light (UVB) radiation play a role? Med Hypotheses. 2006;66(5):993-9.

12. Zijlstra EE, Musa AM, Khalil EA, el-Hassan IM, el-Hassan AM. Post-kala-azar dermal leishmaniasis. Lancet Infec Dis. 2003;3(2):8798.

13. Addy M, Nandy A. Ten years of kala-azar in west Bengal, Part I. Did post-kala-azar dermal leishmaniasis initiate the outbreak in 24-Parganas? Bull World Health Organ. 1992;70(3):341-6. 